Recepción: 28 / 10 / 2017

Aceptación: 24 / 11 / 2017

Publicación: 15/ 12/ 2017

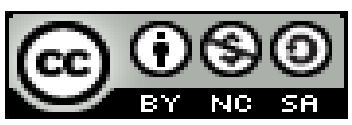

Ciencias técnicas y aplicadas

Artículo de investigación

\title{
Utilización efectiva del enfriador de catalizador
}

Effective use of the catalyst cooler

Uso efetivo do refrigerador do catalisador

\author{
Jairo V. Obando-Perea \\ vladimir.ob91@gmail.com \\ Carlos M. Montaño-Torres \\ cm96478@gmail.com \\ Rita D. Caicedo-Zambrano \\ ritacaicedo@hotmail.com \\ María C. Santos-Falcónez \\ santoscelina97@yahoo.es Nixon \\ E. Proaño-Bone \\ f_porto_27@hotmail.com \\ Edinson E. Castro-Uriarte \\ ezeuriarte@gmail.com
}

\section{Correspondencia: vladimir.ob91@gmail.com}

I. Ingeniero de Petróleos, Docente Universidad Técnica Luis Vargas Torres de Esmeraldas, Esmeraldas, Ecuador.

II. Magister en Ciencias Ambientales, Químico y Farmacéutico, Docente Universidad Técnica Luis Vargas Torres de Esmeraldas, Esmeraldas, Ecuador.

III. Master en Ciencias Especialidad Docencia Universitaria, Licenciada en Ciencias de la Educación Profesora de Segunda Enseñanza Especialidad de Química y Biología, Docente Universidad Técnica Luis Vargas Torres de Esmeraldas, Esmeraldas, Ecuador.

IV. Ingeniera Química, Docente Universidad Técnica Luis Vargas Torres de Esmeraldas, Esmeraldas, Ecuador.

v. Ingeniero Químico, Docente Universidad Técnica Luis Vargas Torres de Esmeraldas, Esmeraldas, Ecuador.

VI. Ingeniero Químico, Docente Universidad Técnica Luis Vargas Torres de Esmeraldas, Esmeraldas, Ecuador. 


\title{
Resumen
}

El equipo enfriador de catalizador, es un intercambiador de calor basado en la utilización de agua fresca, que además de enfriar el catalizador regenerado, debido a la combustión del coque que se produce por la conversión de la carga en el reactor, generará vapor de presión media. El crudo reducido generará una mayor cantidad de residuo de carbón en el regenerador, y que por ende al ser combustionado la cantidad de calor liberado será mayor. El objetivo de este trabajo es determinar la cantidad de calor que se liberará según la variación del \% en peso de crudo reducido y gasóleo, y calcular la cantidad de agua que se producirá en el Catalyst Cooler al mantener el sistema Reactor-Regenerador a temperatura óptima de operación. Para ello se revisa la literatura, se determinan ecuaciones de transferencia de calor y se detallan los equipos. Se realizan los cálculos sobre la cantidad de coque que se producirá en función de la variación del porcentaje en peso de gasóleo y crudo reducido, además de calcular la cantidad de calor que se generará al combustionar el coque que está adherido al catalizador y que ingresará al regenerador para ser descoquizado y ser recirculado hacia el reactor para un nuevo proceso de conversión. Esto aprovecha energía térmica que antes se desperdiciaba provocando impactos ambientales y pérdidas económicas.

Palabras clave: enfriador de catalizador; energía térmica.

\begin{abstract}
The catalyst cooler equipment is a heat exchanger based on the use of fresh water, which in addition to cooling the regenerated catalyst, due to the combustion of the coke that is produced by the conversion of the charge in the reactor, will generate pressure vapor half. The reduced crude will generate a greater amount of carbon residue in the regenerator, and therefore, when combusted, the amount of heat released will be greater. The objective of this work is to determine the amount of heat that will be released according to the variation in $\%$ by weight of reduced crude oil and gas oil, and calculate the amount of water that will be produced in the Catalyst Cooler by keeping the Reactor-Regenerator system at optimum temperature of operation. To do this, the literature is reviewed, heat transfer equations are determined and equipment is detailed. Calculations are made on the amount of coke that will be produced as a function of the variation in the weight percentage of diesel and reduced crude oil, in addition to calculating the amount of
\end{abstract}


heat that will be generated by combustion of the coke that is adhered to the catalyst and that will enter the regenerator to be decoked and recirculated to the reactor for a new conversion process. This takes advantage of thermal energy that was previously wasted causing environmental impacts and economic losses.

Keywords: catalyst cooler; thermal energy.

\section{Resumo}

O equipamento de refrigeração do catalisador é um permutador de calor baseado na utilização de água fresca que, além de arrefecer o catalisador regenerado, devido à combustão da coca que é produzida pela conversão da carga no reator, gerará vapor de pressão média O petróleo reduzido gerará uma maior quantidade de resíduo de carbono no regenerador e, portanto, quando queimado, a quantidade de calor liberada será maior. O objetivo deste trabalho é determinar a quantidade de calor que será liberada de acordo com a variação em\% em peso de petróleo bruto reduzido e gasóleo e calcular a quantidade de água que será produzida no Catalyst Cooler mantendo o sistema Reactor-Regenerator a temperatura ideal de operação. Para fazer isso, a literatura é revista, as equações de transferência de calor são determinadas e o equipamento é detalhado. Os cálculos são feitos sobre a quantidade de coca que será produzida com base na variação da porcentagem em peso de diesel e em bruto reduzido, além de calcular a quantidade de calor que será gerada pela combustão da coca que é aderida ao catalisador e que entrará no regenerador a ser decocado e recirculado ao reator para um novo processo de conversão. Isso aproveita a energia térmica que anteriormente foi desperdiçada causando impactos ambientais e perdas econômicas.

Palavras chave: refrigerador de catalisador; energia térmica.

\section{Introducción}

La Refinería Estatal de Esmeraldas, como empresa refinadora de petróleo, ha mantenido estándares de seguridad aceptables, y su capacidad de refinación es de 110000 bls / día siendo así la que mayor aporta con derivados para abastecimiento interno en el Ecuador. No obstante, se precisa aumentar la producción y a su vez lograr una disminución de costos en generación de vapor. 
La unidad de fraccionamiento catalítico, conocido por sus siglas en inglés como Fluid Catalist Cracking (FCC) o en español como Craqueo Catalítico Fluidizado, que es donde se obtienen los productos livianos de cadena corta con doble enlace (jet fuel, gasolina, LPG, nafta liviana y nafta pesada) debe ser regenerado permanentemente ya que en el reactor se produce la coquización de la superficie activa. Esta operación se realiza en el regenerador por medio de una combustión que genera gran cantidad de energía calórica, hasta la presente esta energía se desperdicia y produce impactos ambientales. En el presente trabajo se diseña un sistema que permita aprovechar esta energía desperdiciada para utilidad en la propia REE.

El Catalist Cooler es un equipo el cual permite retirar el exceso de calor en el regenerador debido al aumento de temperatura, para producir vapor, mediante la utilización de agua. Este equipo fue hecho expresamente para plantas que utilizan un porcentaje de crudo reducido en la carga del reactor de la unidad FCC, pero también se utiliza para introducir propileno al reactor. (Vicente, 2009) (Davison, 2011)

Los componentes de un Catalyst Cooler son:

- Una cámara de combustión orientada verticalmente.

- Una cámara de liberación situado superadyacente y por encima de la cámara de combustión y en comunicación con el mismo.

- Una sección de recogida de catalizador en la parte inferior de la cámara de desprendimiento.

- Un intercambiador de calor de carcasa y tubo de orientación vertical a distancia de las cámaras de combustión y la separación, tiene una entrada de catalizador en una porción superior del lado de la carcasa del intercambiador de calor y una salida de catalizador en una porción inferior del lado de la carcasa del intercambiador de calor.

- Un conducto de catalizador caliente que conecta la parte de recogida de catalizador inferior de la cámara de desprendimiento con la entrada de intercambiador de calor, de tal manera que el catalizador regenerado caliente puede fluir desde la cámara de desprendimiento al intercambiador de calor. 
- Un conducto de catalizador enfriado que conecta la salida de catalizador del intercambiador de calor con una porción inferior de la cámara de combustión, de tal manera que se enfrió el catalizador puede fluir desde el intercambiador de calor a la parte inferior de la cámara de combustión.

- Un conducto de entrada de gas de fluidización conectado a una porción inferior del lado de la carcasa del intercambiador de calor, de tal manera que el gas de fluidificación puede pasar en el lado de la carcasa y mantener un catalizador de lecho fluidizado en el mismo.

- Una válvula de control colocado en el conducto de entrada de gas de fluidización, y un sistema de control que comprende medios para detectar la temperatura en una ubicación seleccionada en la cámara de combustión, medios de control de temperatura que tiene un punto de consigna ajustable de conexión con los medios de detección de temperatura y el desarrollo de una señal de salida, y medios para transmitir la señal de salida a la válvula de control mediante el cual este último se ajusta sensible a la temperatura, con lo que regula el flujo de gas de fluidización en el intercambiador de calor; y los conductos de entrada y salida conectados a los tubos del intercambiador de calor, de tal manera que un fluido de enfriamiento puede fluir a través de los tubos. (USA Patente $n^{\circ} 4434245,2002$ )

Este trabajo presenta como objetivo: Evaluar la entalpía generada por la combustión del coque adherido en el catalizador del reactor de la unidad FCC, para aprovecharlo en la producción de vapor, mediante el equipo Catalist Cooler.

\section{Metodología}

Se hacen los cálculos necesarios y se analizan los resultados.

\section{Desarrollo}

Los cálculos en esta investigación hacen referencia al volumen de coque que se producirá por la reacción y que consecuentemente se adherirá al catalizador limpio que se dirigirá al regenerador para combustionar el coque y de esta manera limpiar el catalizador para ser recirculado al reactor y luego ser utilizado nuevamente. 
Todo este coque que se produce en el regenerador generará una mayor cantidad de calor, el cual será calculado utilizando datos operacionales como son el caudal de coque que se calculó previamente y la capacidad calorífica del coque para obtener un total de calor generado en la combustión.

Debido al calor extra generado existirá un aumento de temperatura, el cual, generaría daños al sistema reactor-regenerador, a más de causar deficiencia al momento de la reacción entre catalizador y el hidrocarburo. Para esto es necesario calcular el volumen de agua que se requerirá ingresar al Catalyst Cooler para mantener la temperatura óptima del regenerador que está entre $\operatorname{los} 420^{\circ} \mathrm{F}$.

Esto se lo logra mediante datos de carbón Conradson de los componentes de la mezcla.

Se asume que es un valor lineal la producción de coque que se tendrá en el regenerador y el cual dependerá el porcentaje de gasóleo y crudo reducido que se tenga respectivamente en la mezcla la cual varía desde el $0 \%$ al $20 \%$ en peso de crudo reducido.

Si la carga a FCC es de $231499.4 \mathrm{lb} / \mathrm{h}$ tenemos que la generación de coque será respectivamente:

Multiplicando la carga por el carbón Conradson de los componentes de la mezcla tenemos que: Coque producido por gasóleo $=0.0607 \% * 231499 \frac{\mathrm{lb}}{\mathrm{h}}=140,52 \mathrm{lb} / \mathrm{h}$

Coque producido por Crudo Reducido $=18,90 * 231 \frac{499.4 l \mathrm{~b}}{\mathrm{~h}}=43753.38 \mathrm{lb} / \mathrm{h}$

Este cálculo se realiza para determinar la cantidad de coque que se producirá al momento de reacción de la carga en el reactor y que se adherirá al catalizador utilizado para acelerar el proceso, para luego ser combustionado en el regenerador para su remoción, teniendo así los caudales de coque que se producirán independientemente para el coque y el crudo reducido.

Se toman las variaciones de los porcentajes más representativos en cuanto a los componentes de la carga, por lo cual tomaremos los siguientes: $20 \%$ gasóleo- $80 \%$ crudo reducido, $90 \%$ gasóleo- 
$10 \%$ crudo reducido y finalmente $97 \%$ gasóleo-3\% crudo reducido que es el valor inicial y el cual se utilizaría sin la implementación del Catalyst Cooler en el Regenerador.

Según el porcentaje en peso de cada uno de los componentes, el coque producido por la mezcla se dará por la siguiente ecuación:

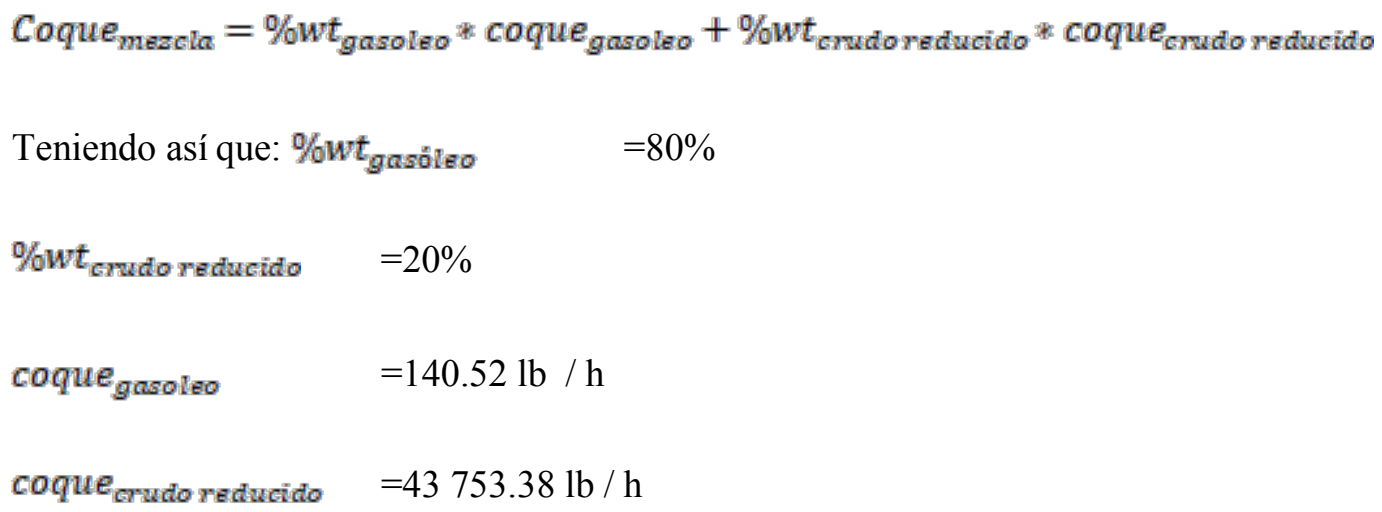

Entonces la masa de coque que se tendrá en el regenerador según la variación de la composición de la carga será respectivamente:

- Mezcla 80\% gasóleo, 20\% Crudo Reducido.

$$
\begin{aligned}
& \text { coque }_{\text {mezcla }(80 \%-20 \%)}=80 \% * 140.5201 \frac{\mathrm{lb}}{\mathrm{h}}+20 \% * 43753.3866 \frac{\mathrm{lb}}{\mathrm{h}} \\
& \text { coque }_{\text {mescla }}=8863.09 \mathrm{lbcoque} / \mathrm{h}
\end{aligned}
$$

- Mezcla 90\% gasóleo, 10\% Crudo Reducido.

$$
\begin{aligned}
& \text { coque }_{\text {mezcla }(90 \%-10 \%)}=90 \% * 140.5201 \frac{\mathrm{lb}}{\mathrm{h}}+10 \% * 43753.3866 \frac{\mathrm{lb}}{\mathrm{h}} \\
& \text { coque }_{\text {mezcla }}=4501.81 \mathrm{lb} \text { coque } / \mathrm{h}
\end{aligned}
$$

- $\quad$ Mezcla 97\% gasóleo, 3\% Crudo Reducido. 
coque $_{\text {mezcla }(9096-1096)}=90 \% * 140.5201 \frac{\mathrm{lb}}{\mathrm{h}}+10 \% * 43753.3866 \frac{\mathrm{lb}}{\mathrm{h}}$

coque $_{\text {mezcla }}=1448.9 \mathrm{lb} \mathrm{coque/}$

En el cálculo anterior se determinó la masa de coque que se producirá respectivamente en el reactor debido a la variación del porcentaje de los componentes que conforman la mezcla.

Del documento Manual de balance de energía en la Unidad FCC de la Refinería Estatal de Esmeraldas se consta que el poder calorífico del coque es $16500 \mathrm{BTU} / \mathrm{lb}$-coque al tener una combustión completa de CO, este valor será constante para cada una de las masas determinadas.

Entonces para determinar los BTU's liberados al combustionar el coque se obtendría de la siguiente forma:

$Q[B T U / h]=$ Poder Calórico ${ }_{\text {coque }}\left[\frac{B T U}{l b_{\text {coque }}}\right] * m_{\text {coque }}\left[\frac{l b_{\text {coque }}}{h}\right]$

Con los datos determinados anteriormente se pueden obtener los siguientes valores:

- Para la mezcla $80 \%$ gasóleo, $20 \%$ crudo reducido se tiene que:

$Q=16500 \frac{B T U}{l b_{\text {coque }}}: 863,1 \mathrm{lb}$ coque

$Q=146241041.6[B T U]$

- Para la mezcla 90\% gasóleo, 10\% crudo reducido se tiene que:

$Q=16500 \frac{B T U}{l b_{\text {coque }}} * 4501,81 l b$ coque

$Q=74279811.91[B T U]$

- Y, para una mezcla de 97\% gasóleo, 3\% crudo reducido tenemos 
$Q=16500 \frac{B T U}{l b_{\text {coque }}} 1448.9 \mathrm{lb}$ coque

$Q=23906951.14[B T U]$

Con los cálculos anteriores obtuvimos el calor que se libera en el regenerador debido a la combustión para limpiar el catalizador gastado y con coque adherido.

La cantidad de calor que se libere dependerá de la masa de coque que se combustione en el regenerador, por ende, mientras mayor porcentaje de crudo reducido tengamos en la mezcla mayor calor se generará.

Sin embargo, a partir de los 23906951.14 [BTU] que equivale a la mezcla del 97\% gasóleo y $3 \%$ crudo reducido es necesaria la utilización del Catalyst Cooler, debido a que este exceso de calor produciría fallas en la regeneración y daños a los equipos debido a las altas temperaturas.

Debido a que el en el Catalyst Cooler se producirá vapor de presión media, la presión de saturación del agua será de 150psi.

Es por lo que para calcular el calor total se utilizaría la siguiente ecuación:

$H_{s}=1119 * 150^{0,01267}$

$H_{s}=1192.34[\mathrm{BTU} / \mathrm{lb}-\mathrm{vapor}]$

El valor de Hs determina la cantidad de calor que se necesita para generar vapor de media en el Catalyst Cooler, y se calcula en base a la presión de saturación a la que se desea generar el vapor.

Y resulta de dividir la cantidad de calor que se generará en el regenerador sobre el calor latente o de vaporización.

Este valor será constante debido a que la presión no variará ya que se necesitará vapor de 150 psi (vapor de media).

Para calcular la masa de agua que ingresa al Catalyst Cooler se utiliza la siguiente ecuación: 
- Para 80\% gasóleo, 20\% crudo reducido:

$$
\begin{aligned}
& m_{\text {vapor }}=\frac{146241041.6[\text { BTU }]}{1192.34\left[\frac{B T U}{\text { lbagua }}\right]} \\
& m_{\text {vapor }}=122650.15[\text { lb vapor } / h]
\end{aligned}
$$

- Para 90\% gasóleo, $10 \%$ crudo reducido:

$$
\begin{gathered}
m_{\text {vapor }}=\frac{74259811.91[B T U]}{1192,34\left[\frac{B T U}{\text { lbagua }}\right]} \\
m_{\text {vapor }}=62297.36[\text { lb vapor } / \mathrm{h}]
\end{gathered}
$$

- Para 97\% gasóleo, 3\% crudo reducido:

$$
\begin{gathered}
m_{\text {vapor }}=\frac{23906951.14[B T U]}{1192,34\left[\frac{B T U}{\text { vbagua }}\right]} \\
m_{\text {vapor }}=20050.40[\mathrm{lb} \text { vapor } / \mathrm{h}]
\end{gathered}
$$

Debido a que la masa es una propiedad que no varía dependiendo de las condiciones tenemos que: La masa de vapor de media presión (150 psi) que se generará en el Catalyst Cooler es igual a la masa de agua que ingresará y que se encuentra a temperatura normal.

A diferencia del volumen de vapor y de agua que si dependerán de las condiciones de presión y temperatura a la que se encuentre.

$$
\text { masa } a_{\text {vapor }}=\text { masa }_{\text {agwa }}
$$

$80 \%$ gasóleo, $20 \%$ crudo reducido

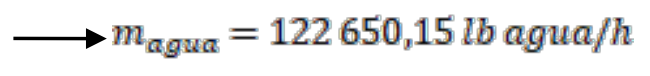

$90 \%$ gasóleo, $10 \%$ crudo reducido

$$
\longrightarrow m_{\text {agwa }}=62297.36[\mathrm{lb} \text { agua } / \mathrm{h}]
$$

97\% gasóleo, $3 \%$ crudo reducido

$$
\longrightarrow m_{a g u a}=20050.40[\mathrm{lb} \text { agua } / \mathrm{h}]
$$


Estos valores determinan la masa de agua que se necesita ingresar al Catalyst Cooler para estabilizar el calor y la temperatura permisible en el regenerador.

Para calcular el volumen de agua se requiere la densidad en $\frac{b b}{m^{g}}$, por lo que es necesario transformar los $0,999 \frac{\mathrm{gr}}{\mathrm{cm}^{\mathrm{g}}}$.

$$
0,999 \frac{\mathrm{gr}}{\mathrm{cm}^{3}} * \frac{1000 \mathrm{~cm}^{3}}{l t} * \frac{1000 \mathrm{lt}}{\mathrm{m}^{3}} * \frac{\mathrm{lb}}{453,6 \mathrm{gr}}=2202,38 \frac{\mathrm{lb}}{\mathrm{m}^{3}}
$$

Con la densidad del agua y la masa en libras se pueden obtener los volúmenes de agua respectivamente: $\rho=\frac{m}{v}$

Despejando tenemos que: $v=\frac{m}{\rho}$

Reemplazando los valores tenemos:

- $80 \%$ gasóleo, $20 \%$ crudo reducido

$\mathrm{m}=122659.1 \mathrm{lb}$-agua

$\rho=2202.38 \frac{l b}{m^{g}}$

$v=\frac{122650.15 \frac{\mathrm{lb} \text { agua }}{h}}{2202.38 \frac{\mathrm{lb}}{\mathrm{m}^{\mathrm{a}}}}$

$v=55.7 m^{3}$ agua $/ h$

- $\quad 90 \%$ gasóleo, $10 \%$ crudo reducido

$\mathrm{m}=62297.35$ lb-agua

$\rho=2202.38 \frac{l b}{m^{3}}$ 


$$
\begin{aligned}
& v=\frac{62297.35 \frac{\mathrm{lb} a g w a}{h}}{2202.38 \frac{\mathrm{lb}}{\mathrm{m}^{\mathrm{a}}}} \\
& v=28.28 \mathrm{~m}^{3} \mathrm{agua} / \mathrm{h}
\end{aligned}
$$

- $\quad 97 \%$ gasóleo, $3 \%$ crudo reducido

$$
\mathrm{m}=122659.1 \text { lb-agua }
$$$$
\rho=2202.38 \frac{w b}{m^{s}}
$$

$v=\frac{20050.40 \frac{\mathrm{lb} a g u a}{h}}{2202.38 \frac{\mathrm{lb}}{\mathrm{m}^{\mathrm{a}}}}$

$$
v=9.10 \mathrm{~m}^{3} \text { agua/h }
$$

El periodo de funcionamiento del Catalyst Cooler es de 8 horas, por tanto el volumen de agua deber ser multiplicado por 8 .

Ya que el agua de enfriamiento es recirculada y debido a que existen pérdidas por condensación y por purgas las cuales se engloban en un $6 \%$ el volumen de agua que se repondrá será de: 472.25 , 239.87, $77.20\left[\mathrm{~m}^{3} /\right.$ día $]$.

Con los volúmenes de agua calculadas podemos realizar los cálculos para determinar las dimensiones de los tanques que abastecerán al Catalyst Cooler teniendo en cuenta un volumen diario de agua y que recircula con pérdidas del 6\%.

Este cálculo se hará por tanteo variando los diámetros del tanque y obteniendo la altura de la ecuación para hallar el volumen de un cilindro el cual es:

$$
\text { volumen del tanque }=\pi * r^{2} * h
$$

Despejando h tenemos que: 


$$
h=\frac{\text { volumen del tanque }}{\pi * r^{2}}
$$

Dónde: h: Altura del tanque. Y r: Radio del tanque.

Para el cálculo de las dimensiones se tomarán en cuenta valores con una mezcla de $80 \%$ de gasóleo y $20 \%$ de crudo reducido y $90 \%$ gasóleo y $10 \%$ crudo reducido, ya que con el 100\% de gasóleo no es necesaria la utilización del Catalyst Cooler.

Entonces tenemos los siguientes datos:

- Para una mezcla $80 \%$ gasóleo, $20 \%$ crudo reducido:

Volumen $\quad=\left[472.25 \mathrm{~m}^{3} /\right.$ día $]$

$\Phi \quad=8.25 \mathrm{~m}$

Reemplazando los valores tenemos que:

$h=\frac{472.25 \mathrm{~m} 3 / \text { día }}{3.141516 * 4.125^{2}}$

$h=8.83 m$

- Para una mezcla 90\% gasóleo, 10\% crudo reducido:

Volumen $\quad=\left[239.87 \mathrm{~m}^{3} /\right.$ día $]$

$\Phi \quad=6.7 \mathrm{~m}$

$h=\frac{239.87 m 3 / d^{\prime} a}{3.141516 * 4,35^{2}}$

$h=6.8 \mathrm{~m}$

- Para una mezcla 97\% gasóleo, 3\% crudo reducido: 


$$
\begin{aligned}
& \text { Volumen } \quad=\left[77.20 \mathrm{~m}^{3} / \text { día }\right] \\
& \Phi \quad=4.5 \mathrm{~m} \\
& h=\frac{77.20 \mathrm{~m} 3 / \text { día }}{3.141516 * 2.25^{2}} \\
& h=4.85 \mathrm{~m}
\end{aligned}
$$

\section{Conclusiones}

- Es de gran importancia para la Refinería Estatal de Esmeraldas la instalación del Catalyst Cooler en la unidad FCC debido a que significa un ahorro en lo que se refiere a gastos en generación de vapor debido a que este equipo generará vapor a partir del exceso de calor que se libere en el regenerador.

- El vapor que se producirá en el Catalyst Cooler será circulado a todas las unidades que requieran calentamiento de los productos, es decir, impliquen transferencias de calor por medio de intercambiadores que utilicen vapor.

- Se realizaron balances de materia y energía en el regenerador de la unidad FCC, para definir el caudal óptimo de agua $\left[\mathrm{m}^{3} /\right.$ día $]$ que debe ingresar al Catalyst Cooler que se convertirá en vapor seco de 150 psi.

- Actualmente la carga de gasóleo al reactor de la unidad FCC contiene un 3\% de crudo reducido proveniente de la destilación atmosférica. Para incrementar la producción de gasolinas se implementará una mezcla de $80 \%$ de gasóleo y $20 \%$ de crudo reducido. Esto provoca mayor coquización del catalizador y mayor calor de combustión en el regenerador, el cual será aprovechado para producir 122650 libras de vapor por hora a 150 psi, esta energía antes se desperdiciaba

- El caudal de agua que ingresará al Catalyst Cooler será de $55.7\left[\mathrm{~m}^{3} / \mathrm{h}\right]$ a la máxima capacidad de crudo reducido. 
- Se calculó el volumen de coque que se producirá en el reactor adherido al catalizador, el cual será combustionado en el regenerador.

- Se pudo determinar que el Catalyst Cooler es necesario a partir de la utilización de un $4 \%$ hasta el $20 \%$ de crudo reducido en la mezcla de la carga de entrada a la FCC ya que en los valores del 0 al 3\% el volumen de agua que se utiliza es despreciable.

- El Catalyst Cooler es un equipo que permite mantener el equilibrio térmico en el sistema Reactor-Regenerador de la unidad FCC por lo que se puede variar la cantidad de agua líquida a ingresar para producir vapor de acuerdo a la cantidad de coque adherida al catalizador en el reactor, en la producción de gasolinas.

- El caudal que ingresa al Catalyst Cooler es $55.7\left[\mathrm{~m}^{3} / \mathrm{h}\right]$ y tras 8 horas de funcionamiento, y más el $6 \%$ de pérdidas se tiene un volumen total de agua de $472.24\left[\mathrm{~m}^{3}\right]$ que debe estar disponible para ingresar al proceso.

\section{Referencias Bibliográficas}

Davison, G. (2011). FCC Conference. En FCC Catalyst Coolers. Munich.

UOP, H. (2002). USA Patente $n^{\circ} 4434245$.

Vicente, P. (2009). Balance de Energía en Calderas. Caracas. 